\title{
Some Nonlinear Stochastic Cauchy Problems with Generalized Stochastic Processes
}

\author{
Victor Dévoué \\ Equipe Analyse Algébrique Non Linéaire, Université des Antilles, Campus de Schoelcher, BP 7209, \\ 97275 Schoelcher Cedex, Martinique
}

Correspondence should be addressed to Victor Dévoué; devoue-vi@orange.fr

Received 4 July 2016; Accepted 25 July 2016

Academic Editor: Ying $\mathrm{Hu}$

Copyright (c) 2016 Victor Dévoué. This is an open access article distributed under the Creative Commons Attribution License, which permits unrestricted use, distribution, and reproduction in any medium, provided the original work is properly cited.

\begin{abstract}
We study some nonlinear stochastic Cauchy problems in the framework of the $(\mathscr{C}, \mathscr{E}, \mathscr{P})$-algebras. We adapt the definitions to this framework. By means of suitable regularizations, we define associated generalized problems. We use our previous results about the wave equation in canonical form to obtain generalized solutions. We compare the generalized solutions with the classical ones when they exist.
\end{abstract}

\section{Introduction}

A possibility in studying stochastic differential equations is to make use of the theory of Colombeau-type generalized functions spaces to overcome the multiplication problem in distribution space. Here, to study some nonlinear stochastic Cauchy problems, we choose to reformulate them correctly in the framework of the $(\mathscr{C}, \mathscr{E}, \mathscr{P})$-algebras of Marti [13 ] in order to show that, following the example of the theory of Colombeau $[4,5]$, these algebras may serve as a tool for treating singular processes in stochastic analysis. Until now, similar studies were made only in Colombeau-type algebras.

A $(\mathscr{C}, \mathscr{E}, \mathscr{P})$-algebra, where $\mathscr{C}$ is overgenerated by a finite set $B$, is always isomorphic to a Colombeau-type algebra but the asymptotic scale of this last algebra, which can be obtained from the generators of $B$, is not explicit and difficult to work with. So, by using the overgeneration, we are able to work with asymptotics which are explicit. Therefore, we consider this framework more convenient.

We adapt the definitions to the framework of the $(\mathscr{C}, \mathscr{E}, \mathscr{P})$-algebras. We interpret generalized stochastic processes on $\mathbb{R}^{2}$ as measurable maps with values in a $(\mathscr{C}, \mathscr{E}, \mathscr{P})$ algebra. Using our previous results about our study on the wave equation in canonical form $[6,7]$, we can prove that some nonlinear non-Lipschitz stochastic Cauchy problems have a unique solution. So this paper completes our research. We find some results similar to those of $[8,9]$ about the solutions in theory of Colombeau algebra.

The paper is organized as follows. In Section 2, we give some definitions and references for stochastic analysis, $(\mathscr{C}, \mathscr{C}, \mathscr{P})$-algebras, and algebras of generalized stochastic processes. In Section 3, we study the following Cauchy problems formally written as

$$
\begin{aligned}
(P): \frac{\partial^{2} U}{\partial x \partial y} & =F(U)+W, \\
\left.U\right|_{\gamma} & =A, \\
\left.\frac{\partial U}{\partial y}\right|_{\gamma} & =B, \\
\left(P^{\prime}\right): \frac{\partial^{2} U}{\partial x \partial y} & =F(U) W, \\
\left.U\right|_{\gamma} & =A, \\
\left.\frac{\partial U}{\partial y}\right|_{\gamma} & =B,
\end{aligned}
$$


where $\gamma$ is a monotonic curve of equation $y=f(x), \gamma$ is not a characteristic curve, $A$ and $B$ are generalized stochastic processes on $\mathbb{R}$, and $W$ is a generalized process on $\mathbb{R}^{2}$. That is, $A, B$, and $W$ are weakly measurable maps of some probability space $(\Omega, \Sigma, \mu)$ with values in the Schwartz distribution space $\mathscr{D}^{\prime}(\mathbb{R})$, respectively, $\mathscr{D}^{\prime}\left(\mathbb{R}^{2}\right)$. The function $F$ is smooth; it can be non-Lipschitz (in $U$ ) but $F$ and all derivatives have polynomial growth.

For $\omega$ fixed, $\omega \in \Omega$, we replace problem $(P)$ (resp., $\left.\left(P^{\prime}\right)\right)$ by a generalized one well-formulated $\left(P(\omega)_{\text {gen }}\right)$ (resp., $\left.\left(P^{\prime}(\omega)_{\text {gen }}\right)\right)$ in a convenient algebra. To do this, we use regularizations and cutoff techniques. We use two parameters. The first parameter regularizes the data and the second one replaces the problem by a family of Lipschitz problems. We show that problem $(P)$ (resp., $\left(P^{\prime}\right)$ ) has a unique solution in some algebras of generalized stochastic processes. Moreover, if problem $(P)$ (resp., $\left(P^{\prime}\right)$ ) admits a solution satisfying appropriate growth estimates on some open subset $O$ of $\mathbb{R}^{2}$, then this solution and the generalized one are equal in a meaning given in Theorem 16.

In Section 4, we are interested in a nonlinear stochastic Cauchy problem with the white noise as initial data:

$$
\begin{aligned}
\left(P_{1}\right): \frac{\partial^{2} U}{\partial x \partial y} & =F(U), \\
\left.U\right|_{\gamma} & =W \\
\left.\frac{\partial U}{\partial y}\right|_{\gamma} & =0
\end{aligned}
$$

where $\gamma$ is the curve of equation $y=f(x), \gamma$ is not a characteristic curve, and $W$ is the white noise on $\mathbb{R}$. The function $F$ is smooth; it can be non-Lipschitz but $F$ and all derivatives have polynomial growth. We treat problem $\left(P_{1}\right)$ in the same way as the previous ones and we study the limiting behavior of the generalized solution.

\section{Algebra of Generalized Stochastic Processes}

2.1. Stochastic Analysis. We refer the reader to [8, 10, 11], for some basic facts from stochastic analysis as construction of white noise and the relation between the white noise and Wiener process on $\mathbb{R}^{d}$.
Let $(\Omega, \Sigma, \mu)$ be a probability space. A weakly measurable map

$$
X: \Omega \longrightarrow \mathscr{D}^{\prime}\left(\mathbb{R}^{d}\right)
$$

is called a generalized stochastic process on $\mathbb{R}^{d}$.

For each fixed test function $\varphi \in \mathscr{D}\left(\mathbb{R}^{d}\right)$, the map $\Omega \rightarrow \mathbb{R}$; $\omega \mapsto\langle X(\omega), \varphi\rangle$ is a random variable. The space of generalized stochastic processes is denoted by $\mathscr{D}_{\Omega}^{\prime}\left(\mathbb{R}^{d}\right)$.

White noise $\dot{W}$ on $\mathbb{R}^{d}$ is constructed as follows.

The probability space is the space of tempered distributions $\Omega=\mathcal{S}^{\prime}\left(\mathbb{R}^{d}\right)$ and $\Sigma$ is the Borel $\sigma$-algebra generated by the weak topology. According to Bochner-Minlos theorem, there is a unique probability measure $\mu$ on $(\Omega, \Sigma)$ such that

$$
\int e^{i\langle X(\omega), \varphi\rangle} d \mu(\omega)=e^{-(1 / 2)\|\varphi\|_{L^{2}\left(\mathbb{R}^{d}\right)}^{2}}
$$

for $\varphi \in \mathcal{S}\left(\mathbb{R}^{d}\right)$.

The white noise $\dot{W}: \Omega \rightarrow \mathscr{D}^{\prime}\left(\mathbb{R}^{d}\right)$ can be define as the identity mapping $\langle X(\omega), \varphi\rangle=\langle\omega, \varphi\rangle, \varphi \in \mathscr{D}\left(\mathbb{R}^{d}\right)$. Remark that $\dot{W}$ is a generalized Gaussian process with mean zero and variance $E\left(\dot{W}(\varphi)^{2}\right)=\|\varphi\|_{L^{2}\left(\mathbb{R}^{d}\right)}^{2}$, where $E$ denotes mathematical expectation. Its covariance is

$$
E(\dot{W}(\varphi) \dot{W}(\psi))=\int_{\mathbb{R}^{d}} \varphi(y) \psi(y) d y .
$$

The white noise on $\mathbb{R}^{d}$ is realized as the $d$-fold distributional derivative of the Wiener process.

\subsection{Algebras of Generalized Functions}

2.2.1. The Presheaves of $(\mathscr{C}, \mathscr{E}, \mathscr{P})$-Algebras. We recall briefly some notions that form the basis of our study [7, 12]. We refer the reader to the references. Take $\mathbb{K}=\mathbb{R}$ or $\mathbb{C}$. Consider

(1) $\Lambda$ a set of indices, $A$ a solid subring of the ring $\mathbb{K}^{\Lambda}$ (that is to say, for any $\left(\left(s_{\lambda}\right)_{\lambda},\left(r_{\lambda}\right)_{\lambda}\right) \in \mathbb{K}^{\Lambda} \times|A|$, with $|A|=\left\{\left(\left|r_{\lambda}\right|\right)_{\lambda}\right.$ : $\left.\left(r_{\lambda}\right)_{\lambda} \in A\right\}$, if $\left(\left|s_{\lambda}\right|\right)_{\lambda} \leq\left(r_{\lambda}\right)_{\lambda}$ (i.e., for any $\left.\lambda,\left|s_{\lambda}\right| \leq r_{\lambda}\right)$, then $\left.\left(s_{\lambda}\right)_{\lambda} \in A\right)$, and $I_{A}$ a solid ideal of $A$;

(2) $\mathscr{E}$ a sheaf of $\mathbb{K}$-topological algebras on a topological space $X$, such that, for any open set $O$ in $X$, the algebra $\mathscr{E}(O)$ is endowed with a family $\mathscr{P}(O)=\left(p_{i}\right)_{i \in I(O)}$ of seminorms satisfying

$$
\forall i \in I(O), \exists(j, k, C) \in I(O) \times I(O) \times \mathbb{R}_{+}^{*}, \forall f, g \in \mathscr{E}(O): p_{i}(f g) \leq C p_{j}(f) p_{k}(g)
$$

Assume that, for any open subsets $\mathrm{O}_{1}, \mathrm{O}_{2}$ of $X$ such that $O_{1} \subset O_{2}$, we have $I\left(O_{1}\right) \subset I\left(O_{2}\right)$ and if $\rho_{1}^{2}$ is the restriction operator $\mathscr{E}\left(O_{2}\right) \rightarrow \mathscr{E}\left(O_{1}\right)$, then, for each $p_{i} \in \mathscr{P}\left(O_{1}\right)$, the seminorm $\widetilde{p}_{i}=p_{i} \circ \rho_{1}^{2}$ extends $p_{i}$ to $\mathscr{P}\left(\mathrm{O}_{2}\right)$.

Assume that, for any family $\mathcal{O}=\left(O_{h}\right)_{h \in H}$ of open subsets of $X$ if $O=\cup_{h \in H} O_{h}$, then, for each $p_{i} \in \mathscr{P}(O), i \in I(O)$, there exists a finite subfamily $O_{1}, \ldots, O_{n(i)}$ of $\mathcal{O}$ and corresponding seminorms $p_{n(j)} \in \mathscr{P}\left(O_{n(j)}\right), 1 \leq j \leq i$, such that, for any $u \in \mathscr{E}(O), p_{i}(u) \leq \sum_{j=1}^{n(i)} p_{n(j)}\left(u_{\mid O_{n(j)}}\right)$.

Set $\mathscr{C}=A / I_{A}$,

$\mathscr{X}_{(A, \mathscr{E}, \mathscr{P})}(O)$

$=\left\{\left(u_{\lambda}\right)_{\lambda} \in[\mathscr{E}(O)]^{\Lambda}: \forall i \in I(O),\left(p_{i}\left(u_{\lambda}\right)\right)_{\lambda} \in|A|\right\}$, 


$$
\begin{aligned}
& \mathcal{N}_{\left(I_{A}, \mathscr{E}, \mathscr{P}\right)}(O) \\
& =\left\{\left(u_{\lambda}\right)_{\lambda} \in[\mathscr{E}(O)]^{\Lambda}: \forall i \in I(O),\left(p_{i}\left(u_{\lambda}\right)\right)_{\lambda} \in\left|I_{A}\right|\right\} .
\end{aligned}
$$

$\mathscr{X}_{(A, \mathscr{E}, \mathscr{P})}$ is a sheaf of subalgebras of the sheaf $\mathscr{E}^{\Lambda}$ and $\mathcal{N}_{\left(I_{A}, \mathscr{E}, \mathscr{P}\right)}$ is a sheaf of ideals of $\mathscr{X}_{(A, \mathscr{E}, \mathscr{P})}$ [13]. The constant sheaf $\mathscr{X}_{(A, \mathbb{K},|\cdot|)} / \mathscr{N}_{\left(I_{A}, \mathbb{K},|\cdot|\right)}$ is the sheaf $\mathscr{C}=A / I_{A}$. We call presheaf of $(\mathscr{C}, \mathscr{E}, \mathscr{P})$-algebra the factor presheaf of algebras $\mathscr{A}=\mathscr{X}_{(A, \mathscr{C}, \mathscr{P})} / \mathscr{N}_{\left(I_{A}, \mathscr{C}, \mathscr{P}\right)}$.

We write $\left[u_{\lambda}\right]$ the class in $\mathscr{A}(O)$ of $\left(u_{\lambda}\right)_{\lambda \in \Lambda} \in \mathscr{X}_{(A, \mathscr{E}, \mathscr{P})}(O)$. We simplify the notations by writing $\mathscr{X}(O)$ (resp., $\mathcal{N}(O)$ ) instead of $\mathscr{X}_{(A, \mathscr{E}, \mathscr{P})}(O)$ (resp., $\mathcal{N}_{\left(I_{A}, \mathscr{E}, \mathscr{P}\right)}(O)$ ).

Overgenerated Rings. Let $B_{p}=\left\{\left(r_{n, \lambda}\right)_{\lambda} \in\left(\mathbb{R}_{+}^{*}\right)^{\Lambda}: n=1, \ldots\right.$, $p\}$ and $B$ be the subset of $\left(\mathbb{R}_{+}^{*}\right)^{\Lambda}$ obtained as rational functions with coefficients in $\mathbb{R}_{+}^{*}$, of elements in $B_{p}$ as variables. Define

$$
\begin{aligned}
A= & \left\{\left(a_{\lambda}\right)_{\lambda} \in \mathbb{K}^{\Lambda} \mid \exists\left(b_{\lambda}\right)_{\lambda} \in B, \exists \lambda_{0} \in \Lambda, \forall \lambda\right. \\
& \left.\prec \lambda_{0}:\left|a_{\lambda}\right| \leq b_{\lambda}\right\} .
\end{aligned}
$$

We say that $A$ is overgenerated by $B_{p}$ ( $A$ is a solid subring of $K^{\Lambda}$ ). If $I_{A}$ is some solid ideal of $A$, we also say that $C=A / I_{A}$ is overgenerated by $B_{p}$.

Relationship with Distribution Theory. Let $O$ be an open subset of $\mathbb{R}^{n}$. If $\left(\varphi_{\varepsilon}\right)_{\varepsilon \in(0,1]}$ is a family of mollifiers $\varphi_{\varepsilon}(x)=\left(1 / \varepsilon^{n}\right) \varphi(x /$ $\varepsilon), x \in \mathbb{R}^{n}, \int \varphi(x) d x=1$ and if $T \in \mathscr{D}^{\prime}\left(\mathbb{R}^{n}\right)$, the convolution product family $\left(T * \varphi_{\varepsilon}\right)_{\varepsilon}$ is a family of smooth functions slowly increasing in $1 / \mathcal{E}$. So, the space of distributions $\mathscr{D}^{\prime}(O)$ can be embedded into $\mathscr{A}(O)$. Taking $\varepsilon$ as component of the multiindex $\lambda \in \Lambda$, we shall choose the subring $A$ overgenerated by some $B_{p}$ of $\left(\mathbb{R}_{+}^{*}\right)^{\Lambda}$ containing the family $(\varepsilon)_{\lambda}$.

Association Process. Consider $O$ an open subset of $X, E$ a sheaf of topological $\mathbb{K}$-vector spaces containing $\mathscr{E}$ as a subsheaf, and $a$ a map from $\Lambda$ to $\mathbb{K}$ such that $(a(\lambda))_{\lambda}=\left(a_{\lambda}\right)_{\lambda}$ is an element of $A$. Assume that

$$
\mathscr{N}(O) \subset\left\{\left(u_{\lambda}\right)_{\lambda} \in \mathscr{X}(O): \lim _{E(O), \Lambda} u_{\lambda}=0\right\} .
$$

We say that $u=\left[u_{\lambda}\right]$ and $v=\left[v_{\lambda}\right] \in \mathscr{E}(O)$ are $a$-E associated if, for each neighborhood $V$ of 0 for the $E$-topology, there exists $\lambda_{0} \in \Lambda$ such that $\lambda \prec \lambda_{0} \Rightarrow a_{\lambda}\left(u_{\lambda}-v_{\lambda}\right) \in V$. We write $u \underset{E(O)}{\stackrel{a}{\sim}} v$ if $\lim _{E(O), \Lambda} a_{\lambda}\left(u_{\lambda}-v_{\lambda}\right)=0$. We define an association process between $u=\left[u_{\lambda}\right]$ and $T \in E(O)$ by $u \sim T \Leftrightarrow$ $\lim _{E(O), \Lambda} u_{\lambda}=T$.

$\mathscr{D}^{\prime}$-Singular Support. Assume that

$$
\begin{aligned}
\mathscr{N}_{\mathscr{D}^{\prime}}^{\mathscr{A}}(O) & =\left\{\left(u_{\lambda}\right)_{\lambda} \in \mathscr{X}(O): \lim _{\mathscr{D}^{\prime}(O), \lambda \rightarrow 0} u_{\lambda}=0\right\} \\
& \supset \mathscr{N}(O) .
\end{aligned}
$$

Let $\mathcal{O}_{D_{\mathscr{A}}^{\prime}}(u)$ be the set of all $x$ having a neighborhood $V$ on which $u$ is associated with a distribution. The $\mathscr{D}^{\prime}$-singular support of $u \in \mathscr{A}(O)$ is the set $\operatorname{singsupp}_{\mathscr{D}^{\prime}}(u)=S_{\mathscr{D}_{\mathscr{A}}^{\prime}}^{\mathscr{A}}(u)=$ $\Omega \backslash \mathcal{O}_{D_{\mathscr{A}}^{\prime}}(u)$.

2.2.2. Generalized Operator and Generalized Restriction Mapping. We denote by $\lambda=(\varepsilon, \eta)$ an element of $\Lambda=\Lambda_{1} \times \Lambda_{2}$, $\Lambda_{1}=\Lambda_{2}=(0,1]$.

Let $O$ be an open subset of $\mathbb{R}^{2}$ and $F_{\eta} \in C^{\infty}(O \times \mathbb{R}, \mathbb{R})$. We say that the algebra $\mathscr{A}(O)$ is stable under the family $\left(F_{\eta}\right)_{\lambda}$ if for all $\left(u_{\lambda}\right)_{\lambda} \in \mathscr{X}(O)$ and $\left(i_{\lambda}\right)_{\lambda} \in \mathcal{N}(O)$ we have $\left(F_{\eta}\left(\cdot, \cdot, u_{\lambda}\right)\right)_{\lambda} \in$ $\mathscr{X}(O),\left(F_{\eta}\left(\cdot, \cdot, u_{\lambda}+i_{\lambda}\right)-F_{\eta}\left(\cdot, \cdot, u_{\lambda}\right)\right)_{\lambda} \in \mathcal{N}(O)$.

If $\mathscr{A}(O)$ is stable under $\left(F_{\eta}\right)_{\lambda}$, for $u=\left[u_{\lambda}\right] \in \mathscr{A}(O)$, $\left[F_{\eta}\left(\cdot, \cdot, u_{\lambda}\right)\right]$ is a well defined element of $\mathscr{A}(O)$ (i.e., not depending on the representative $\left(u_{\lambda}\right)_{\lambda}$ of $u$ ).

The stability condition is verified if the family $\left(F_{\eta}\right)_{\lambda}$ is smoothly tempered, that is to say, if the following two conditions are satisfied:

(i) For each $K \Subset \mathbb{R}^{2}, l \in \mathbb{N}$, and $\left(u_{\lambda}\right)_{\lambda} \in \mathscr{X}(O)$, there is a positive finite sequence $C_{0}, \ldots, C_{l}$, such that $P_{K, l}\left(F_{\eta}\left(\cdot, \cdot, u_{\lambda}\right)\right) \leq \sum_{i=0}^{l} C_{i} P_{K, l}^{i}\left(u_{\lambda}\right)$.

(ii) For each $K \in \mathbb{R}^{2}, l \in \mathbb{N},\left(v_{\lambda}\right)_{\lambda}$, and $\left(u_{\lambda}\right)_{\lambda} \in \mathscr{X}(O)$, there is a positive finite sequence $D_{1}, \ldots, D_{l}$ such that

$$
P_{K, l}\left(F_{\eta}\left(\cdot, \cdot, v_{\lambda}\right)-F_{\eta}\left(\cdot, \cdot, u_{\lambda}\right)\right) \leq \sum_{j=1}^{l} D_{j} P_{K, l}^{j}\left(v_{\lambda}-u_{\lambda}\right) .
$$

Let $F \in C^{\infty}(O \times \mathbb{R}, \mathbb{R})$ and $\left(f_{\eta}\right)_{\eta} \in\left(C^{\infty}(\mathbb{R})\right)^{\Lambda_{1}}$; we define

$$
F_{\eta}(x, y, z)=F\left(x, y, z f_{\eta}(z)\right) .
$$

If $\mathscr{A}(O)$ is stable under $\left(F_{\eta}\right)_{\lambda}$, the operator

$$
\begin{aligned}
\mathscr{F}: \mathscr{A}(O) & \longrightarrow \mathscr{A}(O), \\
u & =\left[u_{\lambda}\right] \longmapsto\left[F_{\eta}\left(\cdot, \cdot, u_{\lambda}\right)\right]
\end{aligned}
$$

is called the generalized operator associated with $F$ via the family $\left(f_{\eta}\right)_{\eta}$.

Consider $f \in C^{\infty}(\mathbb{R})$. We say that $f$ is compatible with second-side restriction if

$$
\begin{aligned}
& \left(u_{\lambda}(\cdot, f(\cdot))\right)_{\lambda} \in \mathscr{X}(\mathbb{R}), \quad \forall\left(u_{\lambda}\right)_{\lambda} \in \mathscr{X}\left(\mathbb{R}^{2}\right), \\
& \left(i_{\lambda}(\cdot, f(\cdot))\right)_{\lambda} \in \mathcal{N}(\mathbb{R}), \quad \forall\left(i_{\lambda}\right)_{\lambda} \in \mathcal{N}\left(\mathbb{R}^{2}\right) .
\end{aligned}
$$

Clearly, if $u=\left[u_{\lambda}\right] \in \mathscr{A}\left(\mathbb{R}^{2}\right)$, then $\left[u_{\lambda}(\cdot, f(\cdot))\right]$ is a well defined element of $\mathscr{A}(\mathbb{R})$.

If the function $f$ is compatible with second-side restriction, the mapping

$$
\begin{aligned}
\mathscr{R}_{f}: \mathscr{A}\left(\mathbb{R}^{2}\right) & \longrightarrow \mathscr{A}(\mathbb{R}), \\
u & =\left[u_{\lambda}\right] \longmapsto\left[u_{\lambda}(\cdot, f(\cdot))\right]
\end{aligned}
$$

is called the generalized second-side restriction mapping associated with $f$.

2.3. The Algebras $\mathscr{A}(O)$ and $\mathscr{A}_{p}(O), p \in \mathbb{N}^{*}$. Set $\mathscr{E}=C^{\infty}$ and $X=\mathbb{R}^{d}$ for $d=1,2$ and $E=\mathscr{D}^{\prime}$ and set $\Lambda$ as a set 
of indices, $\lambda \in \Lambda$. For any open set $O$, in $\mathbb{R}^{d}, \mathscr{E}(O)$ is endowed with the $\mathscr{P}(O)$ topology defined by the family of the seminorms:

$$
\begin{aligned}
P_{K, l}\left(u_{\lambda}\right)= & \sup _{|\alpha| \leq l} P_{K, \alpha}\left(u_{\lambda}\right), \\
& \text { with } P_{K, \alpha}\left(u_{\lambda}\right)=\left\|D^{\alpha} u_{\lambda}(x)\right\|_{L^{\infty}(K)}, K \Subset O
\end{aligned}
$$

and $D^{\alpha}=\partial^{\alpha_{1}+\cdots+\alpha_{d}} /\left(\partial z_{1}^{\alpha_{1}} \cdots \partial z_{d}^{\alpha_{d}}\right)$ for $z=\left(z_{1}, \ldots, z_{d}\right) \in O$, $l \in \mathbb{N}, \alpha=\left(\alpha_{1}, \ldots, \alpha_{d}\right) \in \mathbb{N}^{d}$, and $K \Subset O$ means that $K$ is a compact subset of $O$. Let $A$ be a subring of the ring $\mathbb{R}^{\Lambda}$. We consider a solid ideal $I_{A}$ of $A$. Put

$$
\begin{aligned}
& \mathscr{X}(O)=\left\{\left(u_{\lambda}\right)_{\lambda} \in\left[C^{\infty}(O)\right]^{\Lambda}: \forall K \Subset O, \forall l\right. \\
& \left.\in \mathbb{N},\left(P_{K, l}\left(u_{\lambda}\right)\right)_{\lambda} \in|A|\right\}, \\
& \mathcal{N}(O)=\left\{\left(u_{\lambda}\right)_{\lambda} \in\left[C^{\infty}(O)\right]^{\Lambda}: \forall K \Subset O, \forall l\right. \\
& \left.\in \mathbb{N},\left(P_{K, l}\left(u_{\lambda}\right)\right)_{\lambda} \in\left|I_{A}\right|\right\} .
\end{aligned}
$$

The generalized derivation $D^{\alpha}: u\left(=\left[u_{\varepsilon}\right]\right) \mapsto D^{\alpha} u=\left[D^{\alpha} u_{\varepsilon}\right]$ provides $\mathscr{A}(O)=\mathscr{X}(O) / \mathscr{N}(O)$ with a differential algebraic structure.

Take $p \in \mathbb{N}^{*}$. For any open set $O$, in $\mathbb{R}^{d}, \mathscr{E}(O)=C^{\infty}(O)$ is endowed with the $\mathscr{P}_{p}(O)$ topology defined by the family of the seminorms

$$
\begin{aligned}
N_{K, l}^{p}\left(u_{\lambda}\right) & =\sup _{|\alpha| \leq l} N_{K, \alpha}^{p}\left(u_{\lambda}\right), \\
& \text { with } N_{K, \alpha}^{p}\left(u_{\lambda}\right)=\left\|D^{\alpha} u_{\lambda}(x)\right\|_{L^{p}(K)}, K \Subset O,
\end{aligned}
$$

and $l \in \mathbb{N}, \alpha \in \mathbb{N}^{d}$. Put

$$
\begin{aligned}
\mathscr{X}_{p}(O) & =\left\{\left(u_{\lambda}\right)_{\lambda} \in\left[C^{\infty}(O)\right]^{\Lambda}: \forall K \Subset O, \forall l\right. \\
\in \mathbb{N}, & \left.\left(N_{K, l}^{p}\left(u_{\lambda}\right)\right)_{\lambda} \in|A|\right\}, \\
\mathscr{N}_{p}(O) & =\left\{\left(u_{\lambda}\right)_{\lambda} \in\left[C^{\infty}(O)\right]^{\Lambda}: \forall K \Subset O, \forall l\right. \\
\in \mathbb{N}, & \left.\left(N_{K, l}^{p}\left(u_{\lambda}\right)\right)_{\lambda} \in\left|I_{A}\right|\right\}, \\
\mathscr{A}_{p}(O) & =\frac{\mathscr{X}_{p}(O)}{\mathcal{N}_{p}(O)} .
\end{aligned}
$$

The generalized derivation $D^{\alpha}: u\left(=\left[u_{\varepsilon}\right]\right) \mapsto D^{\alpha} u=\left[D^{\alpha} u_{\varepsilon}\right]$ provides $\mathscr{A}_{p}(O)$ with a differential algebraic structure.

Remark 1. The $N_{K, l}^{2}(p=2)$ norms are bounded by the $P_{K, l}$ norms. We have $\mathscr{A}(O) \subset \mathscr{A}_{2}(O)$.

2.4. Algebras of Generalized Stochastic Processes. Let $O$ be an open set in $\mathbb{R}^{d}$.

Definition 2. A $\mathscr{A}(O)$-generalized stochastic process on a probability space $(\Omega, \Sigma, \mu)$ is a mapping $U: \Omega \rightarrow \mathscr{A}(O)$ such that there is a representing function

$$
u=R_{U}: \Lambda \times O \times \Omega \longrightarrow \mathbb{R}
$$

with the following properties: (i) For fixed $\lambda \in \Lambda$, the map $(x, \omega) \mapsto u(\lambda, x, \omega)$ is jointly measurable on $O \times \Omega$.

(ii) Almost surely in $\omega \in \Omega$, the map $\lambda \mapsto u(\lambda, \cdot, \omega)$ belongs to $\mathscr{X}(O)$ and it is a representative of $U(\omega)$; that is, almost surely in $\omega \in \Omega,\left(U(\omega)_{\lambda}\right)_{\lambda}=(u(\lambda, \cdot, \omega))_{\lambda} \epsilon$ $\mathscr{X}(\mathrm{O})$.

The algebra of generalized stochastic processes is denoted by $\mathscr{A}^{\Omega}(O)$.

Definition 3. A $\mathscr{A}_{2}(0)$-generalized stochastic process on a probability space $(\Omega, \Sigma, \mu)$ is a map $U: \Omega \rightarrow \mathscr{A}_{2}(O)$ such that there is a representing function

$$
u=R_{U}: \Lambda \times O \times \Omega \longrightarrow \mathbb{R}
$$

with the following properties:

(i) For fixed $\lambda \in \Lambda$, the map $(x, \omega) \mapsto u(\lambda, x, \omega)$ is jointly measurable on $O \times \Omega$.

(ii) Almost surely in $\omega \in \Omega$, the map $\lambda \mapsto u(\lambda, \cdot, \omega)$ belongs to $X_{2}(O)$ and it is a representative of $U(\omega)$; that is, almost surely in $\omega \in \Omega,\left(U(\omega)_{\lambda}\right)_{\lambda}=$ $(u(\lambda, \cdot, \omega))_{\lambda} \in \mathscr{X}_{2}(O)$.

The algebra of generalized stochastic processes is denoted by $\mathscr{A}_{2}^{\Omega}(O)$.

Remark 4. Let $\chi \in \mathscr{D}(\mathbb{R})$ having the properties

$$
\begin{gathered}
\int \chi(s) d s=1 ; \\
\int s^{p} \chi(s) d s=0, \quad 1 \leq p \leq 2 ;
\end{gathered}
$$

define $\varphi$ by $\varphi(x, y)=\chi(x) \chi(y)$. Let $V \in \mathscr{D}_{\Omega}^{\prime}\left(\mathbb{R}^{d}\right)$ be a generalized stochastic process. If $\lambda \in \Lambda$, then $V(\omega) * \varphi_{\lambda}$ is measurable with respect to $\omega \in \Omega$ and smooth with respect to $x \in \mathbb{R}^{d}$, hence jointly measurable. Also, $\left(V(\omega) * \varphi_{\lambda}\right)_{\lambda}$ belongs to $\mathscr{X}\left(\mathbb{R}^{d}\right)$. Thus,

$$
R_{V}(\lambda, x, \omega)=\left(V(\omega) * \varphi_{\lambda}\right)(x)=V(\omega)_{\lambda}(x)
$$

qualifies as a representative for a random generalized function. We obtain an imbedding $\tau: \mathscr{D}_{\Omega}^{\prime}\left(\mathbb{R}^{d}\right) \hookrightarrow \mathscr{A}^{\Omega}\left(\mathbb{R}^{d}\right)$.

\section{Nonlinear Stochastic Problems}

3.1. A Nonlinear Stochastic Problem with Additive Generalized Stochastic Process. We consider the Cauchy problem formally written as

$$
\begin{aligned}
(P): \frac{\partial^{2} U}{\partial x \partial y} & =F(U)+W, \\
\left.U\right|_{\gamma} & =A, \\
\left.\frac{\partial U}{\partial y}\right|_{\gamma} & =B,
\end{aligned}
$$


where $\gamma$ is a monotonic curve of equation $y=f(x), \gamma$ is not a characteristic curve, $A, B \in \mathscr{A}^{\Omega}(\mathbb{R})$, and $W \in \mathscr{A}^{\Omega}\left(\mathbb{R}^{2}\right)$ is a $\mathscr{A}\left(\mathbb{R}^{2}\right)$-generalized stochastic process on a probability space $(\Omega, \Sigma, \mu)$. The function $F$ is smooth; it can be non-Lipschitz but $F$ and all derivatives have polynomial growth. Assume that $F(0)=0$. We look for a solution $\left(U: \Omega \rightarrow \mathscr{A}_{2}\left(\mathbb{R}^{2}\right)\right) \in$ $\mathscr{A}_{2}^{\Omega}\left(\mathbb{R}^{2}\right)$ (e.g., we can take $F(U)=-U-U^{3}$ or $F(U)=-U^{3}$ ).

Then, $U$ is a solution to problem $(P)$ if and only if, for any $\omega \in \Omega, U(\omega)$ is solution to the problem $(P(\omega))$ :

$$
\begin{aligned}
(P(\omega)): \frac{\partial^{2} U(\omega)}{\partial x \partial y} & =F(U(\omega))+W(\omega), \\
\left.U(\omega)\right|_{\gamma} & =A(\omega), \\
\left.\frac{\partial U(\omega)}{\partial y}\right|_{\gamma} & =B(\omega) .
\end{aligned}
$$

3.2. A Nonlinear Stochastic Problem with Multiplicative Generalized Stochastic Process. We consider the Cauchy problem formally written as

$$
\begin{aligned}
\left(P^{\prime}\right): \frac{\partial^{2} U}{\partial x \partial y} & =F(U) W, \\
\left.U\right|_{\gamma} & =A, \\
& \left.\frac{\partial U}{\partial y}\right|_{\gamma}=B,
\end{aligned}
$$

where $\gamma$ is a monotonic curve of equation $y=f(x), \gamma$ is not a characteristic curve, $A, B \in \mathscr{A}^{\Omega}(\mathbb{R})$, and $W \in \mathscr{A}^{\Omega}\left(\mathbb{R}^{2}\right)$ is a $\mathscr{A}\left(\mathbb{R}^{2}\right)$-generalized stochastic process on a probability space $(\Omega, \Sigma, \mu)$. The function $F$ is smooth; it can be non-Lipschitz but $F$ and all derivatives have polynomial growth. Assume that $F(0)=0$. We look for a solution $\left(U: \Omega \rightarrow \mathscr{A}_{2}\left(\mathbb{R}^{2}\right)\right) \in$ $\mathscr{A}_{2}^{\Omega}\left(\mathbb{R}^{2}\right)$ (e.g., we can take $F(U)=-U^{3}$ ).

Then, $U$ is a solution to problem $\left(P^{\prime}\right)$ if and only if, for any $\omega \in \Omega, U(\omega)$ is solution to the problem $\left(P^{\prime}(\omega)\right)$ :

$$
\begin{aligned}
\left(P^{\prime}(\omega)\right): \frac{\partial^{2} U(\omega)}{\partial x \partial y} & =F(U(\omega)) W(\omega), \\
\left.U(\omega)\right|_{\gamma} & =A(\omega), \\
\left.\frac{\partial U(\omega)}{\partial y}\right|_{\gamma} & =B(\omega) .
\end{aligned}
$$

3.3. Cutoff Procedure. Let $\left(r_{\eta}\right)_{\eta}$ be in $\mathbb{R}_{*}^{(0,1]}$ such that $r_{\eta}>0$ and $\lim _{\eta \rightarrow 0} r_{\eta}=+\infty$. Set $E_{\eta}=\left[-r_{\eta}, r_{\eta}\right]$.

Consider a family of smooth one-variable functions $\left(h_{\eta}\right)_{\eta}$ such that

$$
\begin{aligned}
& \sup _{z \in I_{\eta}}\left|h_{\eta}(z)\right|=1, \\
& \qquad h_{\eta}(z)= \begin{cases}0, & \text { if }|z| \geq r_{\eta} \\
1, & \text { if }-r_{\eta}+1 \leq z \leq r_{\eta}-1 .\end{cases}
\end{aligned}
$$

Assume that $\partial^{n} h_{\eta} / \partial z^{n}$ is bounded on $E_{\eta}$ for any integer $n$, $n>0$. Set

$$
\sup _{z \in E_{\eta}}\left|\frac{\partial^{n} h_{\eta}}{\partial z^{n}}(z)\right|=M_{n}
$$

Let $\phi_{\eta}(z)=z h_{\eta}(z)$. We approximate the function $F$ by the family of functions $\left(F_{\eta}\right)_{\eta}$ defined by

$$
F_{\eta}(z)=F\left(\phi_{\eta}(z)\right)=F\left(z h_{\eta}(z)\right) .
$$

Assume that $F(0)=0$. Here, function $F$ is smooth; it can be non-Lipschitz but $F$ and all derivatives have polynomial growth. More precisely, we assume the existence of $p \in \mathbb{N}$ such that

$$
\forall l \in \mathbb{N}, \exists c_{l}>0, \sup _{z \in \mathbb{R}}\left|D^{l} F(z)\right| \leq c_{l}(1+|z|)^{p} .
$$

Thus,

$$
\forall l \in \mathbb{N}, \exists \mu_{l}>0, \sup _{z \in \mathbb{R} ;|\alpha| \leq l}\left|D^{\alpha} F_{\eta}(z)\right|=\sup _{|z| \leq r_{\eta} ;|\alpha| \leq l}\left|D^{\alpha} F\left(\phi_{\eta}(z)\right)\right| \leq a_{l}\left(1+r_{\eta}\right)^{p}
$$

So, according to $[7,12], \mathscr{A}(\mathbb{R})$ is stable under the family $\left(F_{\eta}\right)_{\eta}$.

\subsection{Construction of $\mathscr{A}\left(\mathbb{R}^{2}\right)$. Put $U(\omega)=\left[U(\omega)_{\varepsilon, \eta}\right]$ and}

$$
W(\omega)_{\varepsilon, \eta}(x, y)=\left(\phi_{\eta}\left(W(\omega) * \varphi_{\varepsilon}\right)\right)(x, y) .
$$

Define $\varphi$ by $\varphi(x, y)=\chi(x) \chi(y)$ with $\chi \in \mathscr{D}(\mathbb{R})$ having the properties

$$
\begin{gathered}
\int \chi(s) d s=1 \\
\int s^{p} \chi(s) d s=0, \quad 1 \leq p \leq 2 .
\end{gathered}
$$

Consider $\left(\chi_{\varepsilon}\right)_{\varepsilon}$ a family of mollifiers such that $\varkappa_{\varepsilon}(y)=$ $(1 / \varepsilon) \chi(y / \varepsilon)$; then, $\varphi_{\varepsilon}(x, y)=\varkappa_{\varepsilon}(x) \varkappa_{\varepsilon}(y)$. Put $A(\omega)_{\varepsilon}=A(\omega) *$ $\chi_{\varepsilon}$ and $B(\omega)_{\varepsilon}=B(\omega) * \chi_{\varepsilon}$.

We make the following assumptions to generate a convenient $(\mathscr{C}, \mathscr{E}, \mathscr{P})$-algebra adapted to our problem:

$$
\begin{aligned}
& \left(H_{1}\right) \exists p>0, \forall l \in \mathbb{N}, \exists a_{l}>0, \sup _{z \in \mathbb{R} ;|\alpha| \leq l}\left|D^{\alpha} F_{\eta}(z)\right| \leq a_{l}(1+ \\
& \left.r_{\eta}\right)^{p} \text {. } \\
& \left(H_{2}\right) \forall K \Subset \mathbb{R}^{2}, \forall l \in \mathbb{N}, \exists \rho_{l}>0, P_{K, l}\left(W(\omega)_{\varepsilon, \eta}\right) \leq \rho_{K, l}(1+ \\
& \left.r_{\eta}\right)^{p} .
\end{aligned}
$$


$\left(H_{3}\right) \mathscr{C}=A / I_{A}$ is overgenerated by the following elements of $\mathbb{R}_{*}^{(0,1]}:(\varepsilon)_{\varepsilon, \eta},(\eta)_{\varepsilon, \eta},\left(r_{\eta}\right)_{\varepsilon, \eta},\left(\exp r_{\eta}\right)_{\varepsilon, \eta}$.

$\left(H_{4}\right) \mathscr{A}\left(\mathbb{R}^{2}\right)=\mathscr{X}\left(\mathbb{R}^{2}\right) / \mathscr{N}\left(\mathbb{R}^{2}\right)$ is built on $\mathscr{C}$ with $(\mathscr{E}, \mathscr{P})=$ $\left(C^{\infty}\left(\mathbb{R}^{2}\right),\left(P_{K, l}\right)_{K \Subset \mathbb{R}^{2}, l \in \mathbb{N}}\right)$.

$\left(H_{5}\right) \mathscr{A}_{2}\left(\mathbb{R}^{2}\right)=\mathscr{X}_{2}\left(\mathbb{R}^{2}\right) / \mathscr{N}_{2}\left(\mathbb{R}^{2}\right)$ is built on $\mathscr{C}$ with $\left(\mathscr{E}, \mathscr{P}^{\prime}\right)=\left(C^{\infty}\left(\mathbb{R}^{2}\right),\left(N_{K, l}^{2}\right)_{K \in \mathbb{R}^{2}, l \in \mathbb{N}}\right)$.

3.5. Generalized Differential Problems Associated with the Formal Ones. Our goal is to give a meaning to the problems formally written as $(P)$ and $\left(P^{\prime}\right)$.

3.5.1. Generalized Differential Problem Associated with $(P)$. For $\omega$ fixed, the problem associated with $(P(\omega))$ can be written as the well-formulated problem

$$
\begin{aligned}
\left(P(\omega)_{\text {gen }}\right): & \frac{\partial^{2} U(\omega)}{\partial x \partial y}=\mathscr{F}(U(\omega))+\left[W(\omega)_{\varepsilon, \eta}\right] \\
& \mathscr{R}_{f}(U(\omega))=\left[A(\omega)_{\varepsilon}\right] \\
& \mathscr{R}_{f}\left(\frac{\partial U(\omega)}{\partial y}\right)=\left[B(\omega)_{\varepsilon}\right]
\end{aligned}
$$

then,

$$
\begin{aligned}
\frac{\partial^{2} U(\omega)}{\partial x \partial y} & =\left[F_{\eta}(U(\omega))\right]+\left[W(\omega)_{\varepsilon, \eta}\right] \\
\left.U(\omega)\right|_{\gamma} & =\left[A(\omega) * \chi_{\varepsilon}\right] \\
\left.\frac{\partial U(\omega)}{\partial y}\right|_{\gamma} & =\left[B(\omega) * \chi_{\varepsilon}\right] .
\end{aligned}
$$

In terms of representatives, and thanks to the stability and restriction hypothesis, if we can find $U(\omega)_{\varepsilon, \eta} \in C^{\infty}\left(\mathbb{R}^{2}\right)$ verifying

$$
\begin{aligned}
\left(P(\omega)_{(\varepsilon, \eta)}\right): & \frac{\partial^{2} U(\omega)_{\varepsilon, \eta}}{\partial x \partial y}(x, y) \\
= & F_{\eta}\left(U(\omega)_{\varepsilon, \eta}(x, y)\right) \\
& \quad+W(\omega)_{\varepsilon, \eta}(x, y), \\
& U(\omega)_{\varepsilon, \eta}(x, f(x))=A(\omega)_{\varepsilon}(x) \\
= & \left(A(\omega) * \chi_{\varepsilon}\right)(x), \\
& \frac{\partial U(\omega)_{\varepsilon, \eta}}{\partial y}(x, f(x))=B(\omega)_{\varepsilon}(x) \\
= & \left(B(\omega) * \chi_{\varepsilon}\right)(x)
\end{aligned}
$$

and if we can prove that $\left(U(\omega)_{\varepsilon, \eta}\right)_{\varepsilon, \eta} \in \mathscr{X}_{2}\left(\mathbb{R}^{2}\right)$, then $U(\omega)=$ $\left[U(\omega)_{\varepsilon, \eta}\right]$ is a solution of $P(\omega)_{\text {gen }}$.

Let $V(\omega)=\left[V(\omega)_{\varepsilon, \eta}\right]$ be another solution to $P(\omega)_{\text {gen. }}$. The uniqueness of the solution to $P_{\text {gen }}(\omega)$ will be the consequence of $\left(V(\omega)_{\varepsilon, \eta}-U(\omega)_{\varepsilon, \eta}\right)_{\varepsilon, \eta} \in \mathcal{N}\left(\mathbb{R}^{2}\right)$.
Remark 5. Dependence from some regularizing family. The problem $P(\omega)_{\text {gen }}$ itself, and so a solution of it, a priori depends on the family of cutoff functions and, in the case of irregular data, on the family of mollifiers [7].

Remark 6. $F(U)$ is such that

$$
\begin{aligned}
F(U): \Omega & \longrightarrow \mathscr{A}\left(\mathbb{R}^{2}\right), \\
\omega & \longmapsto\left[F_{\eta}\left(U(\omega)_{\varepsilon, \eta}\right)\right], \\
F_{\eta}\left(U(\omega)_{\varepsilon, \eta}\right): \mathbb{R}^{2} & \longrightarrow \mathbb{R}, \\
(x, y) & \longmapsto F_{\eta}\left(U(\omega)_{\varepsilon, \eta}(x, y)\right) .
\end{aligned}
$$

Moreover,

$$
\begin{gathered}
R_{U}=u: \Lambda \times \mathbb{R}^{2} \times \Omega \longrightarrow \mathbb{R} ; \\
(\lambda,(x, y), \omega) \longmapsto U(\omega)_{\varepsilon, \eta}(x, y)=u(\lambda, x, y, \omega),
\end{gathered}
$$

with $\lambda=(\varepsilon, \eta)$.

3.5.2. Generalized Differential Problem Associated with $\left(P^{\prime}\right)$. For $\omega$ fixed, the problem associated with $\left(P^{\prime}(\omega)\right)$ can be written as the well-formulated problem

$$
\begin{aligned}
\left(P_{\text {gen }}^{\prime}(\omega)\right): & \frac{\partial^{2} U(\omega)}{\partial x \partial y}=\mathscr{F}(U(\omega))\left[W(\omega)_{\varepsilon, \eta}\right], \\
& \mathscr{R}_{f}(U(\omega))=\left[A(\omega)_{\varepsilon}\right], \\
& \mathscr{R}_{f}\left(\frac{\partial U(\omega)}{\partial y}\right)=\left[B(\omega)_{\varepsilon}\right] ;
\end{aligned}
$$

then,

$$
\begin{aligned}
\frac{\partial^{2} U(\omega)}{\partial x \partial y} & =\left[F_{\eta}(U(\omega))\right]\left[W(\omega)_{\varepsilon, \eta}\right], \\
\left.U(\omega)\right|_{\gamma} & =\left[A(\omega) * \chi_{\varepsilon}\right], \\
\left.\frac{\partial U(\omega)}{\partial y}\right|_{\gamma} & =\left[B(\omega) * \chi_{\varepsilon}\right] .
\end{aligned}
$$

In terms of representatives, and thanks to the stability and restriction hypothesis, if we can find $U(\omega)_{\varepsilon, \eta} \in C^{\infty}\left(\mathbb{R}^{2}\right)$ verifying

$$
\begin{aligned}
\left(P^{\prime}(\omega)_{(\varepsilon, \eta)}\right): & \frac{\partial^{2} U(\omega)_{\varepsilon, \eta}}{\partial x \partial y}(x, y) \\
& =F_{\eta}\left(U(\omega)_{\varepsilon, \eta}(x, y)\right) W(\omega)_{\varepsilon, \eta}(x, y) \\
& U(\omega)_{\varepsilon, \eta}(x, f(x))=A(\omega)_{\varepsilon}(x) \\
& =\left(A(\omega) * \chi_{\varepsilon}\right)(x) \\
& \frac{\partial U(\omega)_{\varepsilon, \eta}}{\partial y}(x, f(x))=B(\omega)_{\varepsilon}(x) \\
& =\left(B(\omega) * \chi_{\varepsilon}\right)(x)
\end{aligned}
$$


and if we can prove that $\left(U(\omega)_{\varepsilon, \eta}\right)_{\varepsilon, \eta} \in \mathscr{X}_{2}\left(\mathbb{R}^{2}\right)$, then $U(\omega)=$ $\left[U(\omega)_{\varepsilon, \eta}\right]$ is a solution of $\left(P^{\prime}(\omega)_{\mathrm{gen}}\right)$.

\subsection{Generalized Problems}

3.6.1. Solution to the Parametrized Regular Problems. For $\omega$ fixed, we consider the families of regularized problems $\left(P(\omega)_{(\varepsilon, \eta)}\right)$ and $\left(P^{\prime}(\omega)_{(\varepsilon, \eta)}\right)$. We are going to prove that $\left(P(\omega)_{(\varepsilon, \eta)}\right)$ and $\left(P^{\prime}(\omega)_{(\varepsilon, \eta)}\right)$ have a unique smooth solution under assumptions $\left(H_{1}\right)$ and $\left(H_{2}\right)$ and the assumption

$\left(H_{\varepsilon, \eta}\right)$
(a) $f \in C^{\infty}(\mathbb{R}), f^{\prime}>0, f(\mathbb{R})=\mathbb{R}$,
(b) $F_{\eta} \in C^{\infty}(\mathbb{R}, \mathbb{R})$,
(c) $A(\omega)_{\varepsilon}, B(\omega)_{\varepsilon} \in C^{\infty}(\mathbb{R})$.

Following [6], one can prove that $\left(P(\omega)_{(\varepsilon, \eta)}\right)$ is equivalent to the integral formulation

$$
\begin{aligned}
& U(\omega)_{\varepsilon, \eta}(x, y)=U(\omega)_{0, \varepsilon, \eta}(x, y) \\
& -\iint_{D(x, y, f)}\left(F_{\eta}\left(U(\omega)_{\varepsilon, \eta}(\xi, \zeta)\right)\right. \\
& \left.+W(\omega)_{\varepsilon, \eta}(\xi, \zeta)\right) d \xi d \zeta
\end{aligned}
$$

and $\left(P^{\prime}(\omega)_{(\varepsilon, \eta)}\right)$ is equivalent to the integral formulation

$$
\begin{aligned}
& U(\omega)_{\varepsilon, \eta}(x, y)=U(\omega)_{0, \varepsilon, \eta}(x, y) \\
& \quad-\iint_{D(x, y, f)}\left(F_{\eta}\left(U(\omega)_{\varepsilon, \eta}(\xi, \zeta)\right) W(\omega)_{\varepsilon, \eta}(\xi, \zeta)\right) d \xi d \zeta
\end{aligned}
$$

where $U(\omega)_{0, \varepsilon, \eta}(x, y)=\Upsilon(\omega)_{\varepsilon}(y)-\Upsilon(\omega)_{\varepsilon}(f(x))+A(\omega)_{\varepsilon}(x)$ and $\Upsilon(\omega)_{\varepsilon}$ denotes a primitive of $B(\omega)_{\varepsilon} \circ f^{-1}$, with

$$
\begin{aligned}
& D(x, y, f) \\
& = \begin{cases}\left\{(\xi, \zeta): f^{-1}(y) \leq \xi \leq x, y \leq \zeta \leq f(\xi)\right\} & \text { if } y \leq f(x), \\
\left\{(\xi, \zeta): x \leq \xi \leq f^{-1}(y), f(\xi) \leq \zeta \leq y\right\} & \text { if } y \geq f(x) .\end{cases}
\end{aligned}
$$

Theorem 7. Under Assumptions $\left(H_{\varepsilon, \eta}\right),\left(H_{1}\right)$, and $\left(H_{2}\right)$, problem $\left(P(\omega)_{(\varepsilon, \eta)}\right)$ (resp., $\left.\left(P^{\prime}(\omega)_{(\varepsilon, \eta)}\right)\right)$ has a unique solution, $U(\omega)_{\varepsilon, \eta}$, in $C^{\infty}\left(\mathbb{R}^{2}\right)$.

See $[6,12]$ for a detailed proof; replace $u_{\varepsilon, \eta}$ by $U(\omega)_{\varepsilon, \eta}$ and $F_{\eta}\left(x, y, u_{\varepsilon, \eta}(x, y)\right)$ by $F_{\eta}\left(U(\omega)_{\varepsilon, \eta}(x, y)\right)+W(\omega)_{\varepsilon, \eta}(x, y)$ (resp., $\left.F_{\eta}\left(U(\omega)_{\varepsilon, \eta}(x, y)\right) W(\omega)_{\varepsilon, \eta}(x, y)\right)$.

\subsubsection{Solution to the Problems}

Theorem 8. Assume that $U(\omega)_{\varepsilon, \eta}$ is the solution to problem $\left(P(\omega)_{(\varepsilon, \eta)}\right)$ (resp., $\left(P^{\prime}(\omega)_{(\varepsilon, \eta)}\right)$ ); then, problem $\left(P(\omega)_{\text {gen }}\right)$ (resp., $\left.\left(P^{\prime}(\omega)_{\text {gen }}\right)\right)$ has a unique solution $U(\omega)=\left[U(\omega)_{\varepsilon, \eta}\right]$ in $\mathscr{A}\left(\mathbb{R}^{2}\right)$.
$U(\omega)$ is the solution to $\left(P(\omega)_{(\varepsilon, \eta)}\right)$ (resp., $\left.\left(P^{\prime}(\omega)_{(\varepsilon, \eta)}\right)\right)$ if $\left(U(\omega)_{\varepsilon, \eta}\right)_{\varepsilon, \eta} \in \mathscr{X}\left(\mathbb{R}^{2}\right)$; that is,

$$
\left(P_{K, l}\left(U(\omega)_{\varepsilon, \eta}\right)\right)_{\varepsilon, \eta} \in A, \quad \forall K \Subset \mathbb{R}^{2}, \forall l \in \mathbb{N} .
$$

The proof follows the same steps as the existence results which can be found in [12], replacing $u_{\varepsilon, \eta}$ by $U(\omega)_{\varepsilon, \eta}$ and $F_{\eta}\left(x, y, u_{\varepsilon, \eta}(x, y)\right)$ by $F_{\eta}\left(U(\omega)_{\varepsilon, \eta}(x, y)\right)+W(\omega)_{\varepsilon, \eta}(x, y)$ (resp., $\left.F_{\eta}\left(U(\omega)_{\varepsilon, \eta}(x, y)\right) W(\omega)_{\varepsilon, \eta}(x, y)\right)$. An induction process on the order of the successive derivatives shows that $\left(U(\omega)_{\varepsilon, \eta}\right)_{\varepsilon, \eta}$ belongs to $\mathscr{X}\left(\mathbb{R}^{2}\right)$. The Gronwall lemma is essential tool for the uniqueness.

Theorem 9. Assume that $U(\omega)_{\varepsilon, \eta}$ is the solution to problem $\left(P(\omega)_{(\varepsilon, \eta)}\right)$ (resp., $\left(P^{\prime}(\omega)_{(\varepsilon, \eta)}\right)$ ); then, problem $\left(P(\omega)_{g e n}\right)$ (resp., $\left.\left(P^{\prime}(\omega)_{\text {gen }}\right)\right)$ has a unique solution $U(\omega)=\left[U(\omega)_{\varepsilon, \eta}\right]$ in $\mathscr{A}_{2}\left(\mathbb{R}^{2}\right)$.

Proof. $U(\omega)$ is the solution to $\left(P(\omega)_{(\varepsilon, \eta)}\right)$ if $\left(U(\omega)_{\varepsilon, \eta}\right)_{\varepsilon, \eta} \in$ $\mathscr{X}_{2}\left(\mathbb{R}^{2}\right)$. We shall prove that

$$
\left(N_{K, l}^{2}\left(U(\omega)_{\varepsilon, \eta}\right)\right)_{\varepsilon, \eta} \in A, \quad \forall K \Subset \mathbb{R}^{2}, \forall l \in \mathbb{N} .
$$

But

$$
\begin{aligned}
& \left\|D^{\alpha}\left(U(\omega)_{\varepsilon, \eta}\right)\right\|_{L^{2}(K)} \\
& \quad \leq(\mu(K))^{1 / 2}\left\|D^{\alpha}\left(U(\omega)_{\varepsilon, \eta}\right)\right\|_{L^{\infty}(K)}
\end{aligned}
$$

and, as $\left(U(\omega)_{\varepsilon, \eta}\right)_{\varepsilon, \eta} \in \mathscr{X}\left(\mathbb{R}^{2}\right)$, we have $\left(\left\|D^{\alpha}\left(U(\omega)_{\varepsilon, \eta}\right)\right\|_{\infty}\right)_{\varepsilon, \eta} \in$ $A$. Thus,

$$
\left(\left\|D^{\alpha}\left(U(\omega)_{\varepsilon, \eta}\right)\right\|_{L^{2}(K)}\right)_{\varepsilon, \eta}=\left(N_{K, l}^{2}\left(U(\omega)_{\varepsilon, \eta}\right)\right)_{\varepsilon, \eta} \in A .
$$

So $U(\omega) \in \mathscr{A}_{2}\left(\mathbb{R}^{2}\right)$ and it is the solution to problem $\left(P(\omega)_{\text {gen }}\right)$ in $\mathscr{A}_{2}\left(\mathbb{R}^{2}\right)$. Set

$$
\begin{gathered}
U: \Omega \longrightarrow \mathscr{A}_{2}\left(\mathbb{R}^{2}\right), \\
\omega \longmapsto U(\omega) .
\end{gathered}
$$

Then, $U \in \mathscr{A}_{2}^{\Omega}\left(\mathbb{R}^{2}\right)$.

Theorem 10. The mapping $U$ is the solution to problem $(P)$ (resp., $\left(P^{\prime}\right)$ ) and it is almost surely unique in $\mathscr{A}_{2}^{\Omega}\left(\mathbb{R}^{2}\right)$.

Proof. Since $U(\omega)$ is the unique solution to problem $\left(P(\omega)_{\text {gen }}\right)$ in $\mathscr{A}_{2}\left(\mathbb{R}^{2}\right)$, then almost surely in $\omega \in \Omega$, the map $\lambda \mapsto$ $R_{U}(\lambda,(\cdot, \cdot), \omega)=U(\omega)_{\lambda},(\lambda=(\varepsilon, \eta))$, belongs to $\mathscr{X}_{2}\left(\mathbb{R}^{2}\right)$ and it is a representative of $U(\omega)$ (i.e., $\left.U(\omega)=\left[U(\omega)_{\lambda}\right]\right)$. For fixed $\lambda=(\varepsilon, \eta) \in \Lambda$, the map

$$
((x, y), \omega) \longmapsto R_{U}(\lambda,(x, y), \omega)=U(\omega)_{\lambda}(x, y)=u_{\lambda}((x, y), \omega)
$$


is jointly measurable on $\mathbb{R}^{2} \times \Omega$. So $U$ is the solution to problem $(P)$ almost surely unique in $\mathscr{A}_{2}^{\Omega}\left(\mathbb{R}^{2}\right)$.

\subsection{The Regularized and the Nonregularized Problems}

Remark 11. The generalized function represented by the family of solutions to the regularized problems $\left(P(\omega)_{(\varepsilon, \eta)}\right)$ (resp., $\left.\left(P^{\prime}(\omega)_{(\varepsilon, \eta)}\right)\right)$ is defined from the integral representation. Thus, we are going to study the relationship between this generalized function and the classical solutions to $\left(P(\omega)_{\text {gen }}\right)$ (resp., $\left.\left(P^{\prime}(\omega)_{(\varepsilon, \eta)}\right)\right)$, when they exist, on a domain $O$ such that $\forall(x, y) \in O, D(x, y, f) \subset O$. This justifies choosing $O=] a, b[\times] f(a), f(b)\left[\right.$ when $(a, b) \in \mathbb{R}^{2}$ with $a<b$.

If problem $\left(P(\omega)_{\text {gen }}\right)$ (resp., $\left.\left(P^{\prime}(\omega)_{\text {gen }}\right)\right)$ has a smooth solution $v$ on $O$, then, necessarily, we have $O \subset \mathbb{R}^{2} \mid$ $\operatorname{singsupp}(U(\omega))$.

Let us recall that there exists a canonical sheaf embedding of $C^{\infty}$ into $\mathscr{A}$, through the morphism of algebra

$$
\begin{aligned}
\sigma_{O}: C^{\infty}(O) & \longrightarrow \mathscr{A}(O), \\
g & \longmapsto\left[g_{\varepsilon}\right],
\end{aligned}
$$

where $O$ is any open subset of $\mathbb{R}^{2}$ and $g_{\varepsilon}=g$. The presheaf $\mathscr{A}$ allows defining restriction and, as usually, we denote by $\left.u\right|_{O}$ the restriction on $O$ of $U \in \mathscr{A}\left(\mathbb{R}^{2}\right)$.

Theorem 12. Let $O$ be an open subset of $\mathbb{R}^{2}$ such that

$$
O \subset \mathbb{R}^{2} \backslash \operatorname{singsupp}(U(\omega)) .
$$

Assume that $O=\bigcup_{\eta} O_{\eta}$ when $\left(O_{\eta}\right)_{\eta}$ is an increasing family of open subsets of $\mathbb{R}^{2}$ such that $\left.O_{\eta}=\right] a_{\eta}, b_{\eta}[\times] f\left(a_{\eta}\right), f\left(b_{\eta}\right)[$ when $\left(a_{\eta}, b_{\eta}\right) \in \mathbb{R}^{2}$ with $a_{\eta}<b_{\eta}$. Assume that the nonregularized problem $\left(P(\omega)_{\text {gen }}\right)$ (resp., $\left.\left(P^{\prime}(\omega)_{\text {gen }}\right)\right)$ has a smooth solution $V(\omega)$ on $O$ such that $\sup _{(x, y) \in O_{\eta}}|V(\omega)(x, y)|<$ $r_{\eta}-1$ for any $\eta$. Let $U(\omega) \in \mathscr{A}\left(\mathbb{R}^{2}\right)$ be the generalized function represented by the family $\left(U(\omega)_{\varepsilon, \eta}\right)_{\varepsilon, \eta}$ of solutions to $\left(P(\omega)_{(\varepsilon, \eta)}\right)$ (resp., $\left.\left(P^{\prime}(\omega)_{(\varepsilon, \eta)}\right)\right)$. Then, $\sigma_{O}(V(\omega))=\left.U(\omega)\right|_{O}$.

We refer the reader to [12] for a detailed proof.

We can say that the solution $U=[U(\omega)]$ to the regularized problem coincides with the solution to the nonregularized equation on $O$.

3.8. A Special Case. We consider the Cauchy problem formally written as

$$
\begin{aligned}
(S): \frac{\partial^{2} U}{\partial x \partial y}=W, \\
\left.U\right|_{\gamma}=A, \\
\left.\frac{\partial U}{\partial y}\right|_{\gamma}=B,
\end{aligned}
$$

where $\gamma$ is a monotonic curve of equation $y=f(x), \gamma$ is not a characteristic curve, $A, B \in \mathscr{A}^{\Omega}(\mathbb{R})$, and $W \in \mathscr{A}^{\Omega}\left(\mathbb{R}^{2}\right)$ is a
$\mathscr{A}\left(\mathbb{R}^{2}\right)$-generalized stochastic process on a probability space $(\Omega, \Sigma, \mu)$.

This problem coincides with problem $(P)$ for $F=0$ and with problem $\left(P^{\prime}\right)$ for $F=1$. We obtain the solution $U$ to problem $(S) \cdot U(\omega)=\left[U(\omega)_{\varepsilon, \eta}\right]$ in $\mathscr{A}_{2}\left(\mathbb{R}^{2}\right)$ is defined, with the previous notations, by

$$
\begin{aligned}
U(\omega)_{\varepsilon, \eta}(x, y)= & U(\omega)_{0, \varepsilon, \eta}(x, y) \\
& -\iint_{D(x, y, f)} W(\omega)_{\varepsilon, \eta}(\xi, \zeta) d \xi d \zeta .
\end{aligned}
$$

\section{A Nonlinear Stochastic Cauchy Problem with the White Noise as Data}

We consider the Cauchy problems formally written as

$$
\begin{aligned}
\left(P_{1}\right): \frac{\partial^{2} U}{\partial x \partial y} & =F(U), \\
\left.U\right|_{\gamma} & =W, \\
\left.\frac{\partial U}{\partial y}\right|_{\gamma} & =0, \\
\left(P_{2}\right): \frac{\partial^{2} V}{\partial x \partial y} & =0, \\
\left.V\right|_{\gamma} & =W, \\
\left.\frac{\partial V}{\partial y}\right|_{\gamma} & =0,
\end{aligned}
$$

where $\gamma$ is a monotonic curve of equation $y=f(x), \gamma$ is not a characteristic curve, and $W \in \mathscr{A}^{\Omega}(\mathbb{R})$ is the white noise on $\mathbb{R}$. The function $F$ is smooth; it can be non-Lipschitz but $F$ and all derivatives have polynomial growth. Assume that $F(0)=0$. We look for a solution $\left(U: \Omega \rightarrow \mathscr{A}\left(\mathbb{R}^{2}\right)\right) \in \mathscr{A}^{\Omega}\left(\mathbb{R}^{2}\right)$ and $\left(V: \Omega \rightarrow \mathscr{A}\left(\mathbb{R}^{2}\right)\right) \in \mathscr{A}^{\Omega}\left(\mathbb{R}^{2}\right)$.

$U$ is a solution to problem $\left(P_{1}\right)$ if and only if, for any $\omega \in$ $\Omega, U(\omega)$ is a solution to the formally problem

$$
\begin{aligned}
\left(P_{1}(\omega)\right): \frac{\partial^{2} U(\omega)}{\partial x \partial y} & =F(U(\omega)), \\
\left.U(\omega)\right|_{\gamma} & =W(\omega), \\
\left.\frac{\partial U(\omega)}{\partial y}\right|_{\gamma} & =0 .
\end{aligned}
$$

$V$ is a solution to problem $\left(P_{2}\right)$ if and only if, for any $\omega \in \Omega$, $V(\omega)$ is a solution to the formally problem

$$
\begin{aligned}
\left(P_{2}(\omega)\right): \frac{\partial^{2} V(\omega)}{\partial x \partial y} & =0, \\
\left.V(\omega)\right|_{\gamma} & =W(\omega), \\
\left.\frac{\partial V(\omega)}{\partial y}\right|_{\gamma} & =0 .
\end{aligned}
$$


We consider the same hypotheses and we take the same spaces $\mathscr{A}\left(\mathbb{R}^{2}\right)$ and $\mathscr{A}_{2}\left(\mathbb{R}^{2}\right)$ built for problems $(P)$ and $\left(P^{\prime}\right)$.

4.1. A Generalized Differential Problem Associated with the Formal One. Our goal is to give a meaning to the problem formally written as $\left(P_{1}(\omega)\right)$. For $\omega$ fixed, the problem associated with $\left(P_{1}(\omega)\right)$ can be written as the well-formulated problem

$$
\begin{aligned}
\left(P_{\text {1gen }}(\omega)\right): & \frac{\partial^{2} U(\omega)}{\partial x \partial y}=\mathscr{F}(U(\omega)), \\
& \mathscr{R}_{f}(U(\omega))=\left[W(\omega)_{\varepsilon}\right] \\
& \mathscr{R}_{f}\left(\frac{\partial U(\omega)}{\partial y}\right)=0
\end{aligned}
$$

then,

$$
\begin{aligned}
\frac{\partial^{2} U(\omega)}{\partial x \partial y} & =\left[F_{\eta}(U(\omega))\right], \\
\left.U(\omega)\right|_{\gamma} & =\left[W(\omega) * \chi_{\varepsilon}\right], \\
\left.\frac{\partial U(\omega)}{\partial y}\right|_{\gamma} & =0 .
\end{aligned}
$$

The problem associated with $\left(P_{2}(\omega)\right)$ can be written as the well-formulated problem

$$
\begin{aligned}
\left(P_{\text {2gen }}(\omega)\right): & \frac{\partial^{2} V(\omega)}{\partial x \partial y}=0, \\
& \mathscr{R}_{f}(V(\omega))=\left[W(\omega)_{\varepsilon}\right], \\
& \mathscr{R}_{f}\left(\frac{\partial V(\omega)}{\partial y}\right)=0 ;
\end{aligned}
$$

then,

$$
\begin{aligned}
\frac{\partial^{2} V(\omega)}{\partial x \partial y} & =0, \\
\left.V(\omega)\right|_{\gamma} & =\left[W(\omega) * \chi_{\varepsilon}\right], \\
\left.\frac{\partial V(\omega)}{\partial y}\right|_{\gamma} & =0 .
\end{aligned}
$$

In terms of representatives, and thanks to the stability and restriction hypothesis, if we can find $U(\omega)_{\varepsilon, \eta} \in C^{\infty}\left(\mathbb{R}^{2}\right)$ verifying

$$
\begin{aligned}
\left(P_{1}(\omega)_{(\varepsilon, \eta)}\right): & \frac{\partial^{2} U(\omega)_{\varepsilon, \eta}}{\partial x \partial y}(x, y) \\
& =F_{\eta}\left(U(\omega)_{\varepsilon, \eta}(x, y)\right), \\
& U(\omega)_{\varepsilon, \eta}(x, f(x)) \\
& =\left(W(\omega) * \chi_{\varepsilon}\right)(x), \\
& \frac{\partial U(\omega)_{\varepsilon, \eta}}{\partial y}(x, f(x))=0
\end{aligned}
$$

and if we can prove that $\left(U(\omega)_{\varepsilon, \eta}\right)_{\varepsilon, \eta} \in \mathscr{X}_{2}\left(\mathbb{R}^{2}\right)$, then $U(\omega)=$ $\left[U(\omega)_{\varepsilon, \eta}\right]$ is a solution of $\left(P_{1}(\omega)_{\text {gen }}\right)$.

We have $\left[V(\omega)_{\varepsilon, \eta}\right]=W(\omega) * \chi_{\varepsilon}$; then, $V(\omega)_{\varepsilon, \eta}(x, y)=$ $\left(W(\omega) * \chi_{\varepsilon}\right)(x)$.

\subsection{Generalized Problem}

4.2.1. Solution to the Parametrized Regular Problem. For $\omega$ fixed, we consider the family of regularized problems $\left(P_{1}(\omega)_{(\varepsilon, \eta)}\right)$. We are going to prove that $\left(P_{1}(\omega)_{(\varepsilon, \eta)}\right)$ has a unique smooth solution under the following assumptions:

$$
\begin{aligned}
& \left(H_{\varepsilon, \eta}\right) \\
& \quad \text { (a) } f \in C^{\infty}(\mathbb{R}), f^{\prime}>0, f(\mathbb{R})=\mathbb{R}, \\
& \text { (b) } F_{\eta} \in C^{\infty}(\mathbb{R}, \mathbb{R}), \\
& \text { (c) } W(\omega)_{\varepsilon} \in C^{\infty}(\mathbb{R}) . \\
& \left(H_{1}\right) \exists p>0, \forall l \in \mathbb{N}, \exists a_{l}>0, \sup _{z \in \mathbb{R} ;|\alpha| \leq l}\left|D^{\alpha} F_{\eta}(z)\right| \leq \\
& a_{l}\left(1+r_{\eta}\right)^{p} .
\end{aligned}
$$

Following [6], one can prove that $\left(P_{1}(\omega)_{(\varepsilon, \eta)}\right)$ is equivalent to the integral formulation

$$
\begin{aligned}
& U(\omega)_{\varepsilon, \eta}(x, y) \\
& =U(\omega)_{0, \varepsilon, \eta}(x, y) \\
& \quad-\iint_{D(x, y, f)} F_{\eta}\left(U(\omega)_{\varepsilon, \eta}(\xi, \zeta)\right) d \xi d \zeta,
\end{aligned}
$$

where $U(\omega)_{0, \varepsilon, \eta}(x, y)=\left(W(\omega) * \chi_{\varepsilon}\right)(x)$, with

$$
\begin{aligned}
& D(x, y, f) \\
& = \begin{cases}\left\{(\xi, \zeta): f^{-1}(y) \leq \xi \leq x, y \leq \zeta \leq f(\xi)\right\} & \text { if } y \leq f(x), \\
\left\{(\xi, \zeta): x \leq \xi \leq f^{-1}(y), f(\xi) \leq \zeta \leq y\right\} & \text { if } y \geq f(x) .\end{cases}
\end{aligned}
$$

Theorem 13. Under Assumptions $\left(H_{\varepsilon, \eta}\right)$ and $\left(H_{1}\right)$, problem $\left(P_{1}(\omega)_{(\varepsilon, \eta)}\right)$ has a unique solution, $U(\omega)_{\varepsilon, \eta}$, in $C^{\infty}\left(\mathbb{R}^{2}\right)$.

We refer the reader to $[6,12]$ for a detailed proof (replace $u_{\varepsilon, \eta}$ by $U(\omega)_{\varepsilon, \eta}$ and $F_{\eta}\left(x, y, u_{\varepsilon, \eta}(x, y)\right)$ by $\left.F_{\eta}\left(U(\omega)_{\varepsilon, \eta}(x, y)\right)\right)$.

\subsubsection{Solution to $\left(P_{1}\right)$}

Theorem 14. Assume that $U(\omega)_{\varepsilon, \eta}$ is the solution to problem $\left(P_{1}(\omega)_{(\varepsilon, \eta)}\right)$; then, problem $\left(P_{1}(\omega)_{g e n}\right)$ has a unique solution $U(\omega)=\left[U(\omega)_{\varepsilon, \eta}\right]$ in $\mathscr{A}\left(\mathbb{R}^{2}\right)$.

See [12] for a detailed proof (replace $u_{\varepsilon, \eta}$ by $U(\omega)_{\varepsilon, \eta}$ and $F_{\eta}\left(x, y, u_{\varepsilon, \eta}(x, y)\right)$ by $\left.F_{\eta}\left(U(\omega)_{\varepsilon, \eta}(x, y)\right)\right)$.

Theorem 15. Assume that $U(\omega)_{\varepsilon, \eta}$ is the solution to problem $\left(P_{1}(\omega)_{(\varepsilon, \eta)}\right)$; then, problem $\left(P_{1}(\omega)_{\text {gen }}\right)$ has a unique solution $U(\omega)=\left[U(\omega)_{\varepsilon, \eta}\right]$ in $\mathscr{A}_{2}\left(\mathbb{R}^{2}\right)$.

Proof. $U(\omega)$ is the solution to $\left(P_{1}(\omega)_{(\varepsilon, \eta)}\right)$ if $\left(U(\omega)_{\varepsilon, \eta}\right)_{\varepsilon, \eta} \in$ $\mathscr{X}_{2}\left(\mathbb{R}^{2}\right)$. We shall prove that

$$
\left(N_{K, l}^{2}\left(U(\omega)_{\varepsilon, \eta}\right)\right)_{\varepsilon, \eta} \in A, \quad \forall K \Subset \mathbb{R}^{2}, \forall l \in \mathbb{N} .
$$


But

$$
\begin{aligned}
& \left\|D^{\alpha}\left(U(\omega)_{\varepsilon, \eta}\right)\right\|_{L^{2}(K)} \\
& \quad \leq(\mu(K))^{1 / 2}\left\|D^{\alpha}\left(U(\omega)_{\varepsilon, \eta}\right)\right\|_{L^{\infty}(K)}
\end{aligned}
$$

and, as $\left(U(\omega)_{\varepsilon, \eta}\right)_{\varepsilon, \eta} \in \mathscr{X}\left(\mathbb{R}^{2}\right)$, we have $\left(\left\|D^{\alpha}\left(U(\omega)_{\varepsilon, \eta}\right)\right\|_{\infty}\right)_{\varepsilon, \eta} \in$ $A$. Thus,

$$
\left(\left\|D^{\alpha}\left(U(\omega)_{\varepsilon, \eta}\right)\right\|_{L^{2}(K)}\right)_{\varepsilon, \eta}=\left(N_{K, l}^{2}\left(U(\omega)_{\varepsilon, \eta}\right)\right)_{\varepsilon, \eta} \in A .
$$

So $U(\omega) \in \mathscr{A}_{2}\left(\mathbb{R}^{2}\right)$ and it is the solution to problem $\left(P_{1}(\omega)_{\text {gen }}\right)$ in $\mathscr{A}_{2}\left(\mathbb{R}^{2}\right)$. Set

$$
\begin{gathered}
U: \Omega \longrightarrow \mathscr{A}_{2}\left(\mathbb{R}^{2}\right), \\
\omega \longmapsto U(\omega) .
\end{gathered}
$$

Then, $U \in \mathscr{A}_{2}^{\Omega}\left(\mathbb{R}^{2}\right)$.

Theorem 16. The mapping $U$ is the solution to problem $\left(P_{1}\right)$ and it is almost surely unique in $\mathscr{A}_{2}^{\Omega}\left(\mathbb{R}^{2}\right)$.

Proof. Since $U(\omega)$ is the unique solution to problem $\left(P_{1}(\omega)_{\text {gen }}\right)$ in $\mathscr{A}_{2}\left(\mathbb{R}^{2}\right)$, then almost surely in $\omega \in \Omega$, the map $\lambda \mapsto R_{U}(\lambda,(\cdot, \cdot), \omega)=U(\omega)_{\lambda},(\lambda=(\varepsilon, \eta))$, belongs to $\mathscr{X}_{2}\left(\mathbb{R}^{2}\right)$ and it is a representative of $U(\omega)$ (i.e., $U(\omega)=\left[U(\omega)_{\lambda}\right]$ ). For fixed $\lambda=(\varepsilon, \eta) \in \Lambda$, the map

$$
((x, y), \omega) \longmapsto R_{U}(\lambda,(x, y), \omega)=U(\omega)_{\lambda}(x, y)
$$

is jointly measurable on $\mathbb{R}^{2} \times \Omega$. So $U$ is the solution to problem $\left(P_{1}\right)$ almost surely unique in $\mathscr{A}_{2}^{\Omega}\left(\mathbb{R}^{2}\right)$.

Theorem 17. Let $O$ be an open subset of $\mathbb{R}^{2}$ such that

$$
O \subset \mathbb{R}^{2} \backslash \operatorname{singsupp}(U(\omega)) .
$$

Assume that $\mathrm{O}=\bigcup_{\eta} O_{\eta}$ when $\left(O_{\eta}\right)_{\eta}$ is an increasing family of open subsets of $\mathbb{R}^{2}$ such that $\left.O_{\eta}=\right] a_{\eta}, b_{\eta}[\times] f\left(a_{\eta}\right), f\left(b_{\eta}\right)[$ when $\left(a_{\eta}, b_{\eta}\right) \in \mathbb{R}^{2}$ with $a_{\eta}<b_{\eta}$. Assume that the nonregularized problem has a smooth solution $V(\omega)$ on $O$ such that $\sup _{(x, y) \in O_{\eta}}|V(\omega)(x, y)|<r_{\eta}-1$ for any $\eta$. Let $U(\omega) \in$ $\mathscr{A}\left(\mathbb{R}^{2}\right)$ be the generalized function represented by the family $\left(U(\omega)_{\varepsilon, \eta}\right)_{\varepsilon, \eta}$ of solutions to $\left(P_{1}(\omega)_{(\varepsilon, \eta)}\right)$. Then, $\sigma_{O}(V(\omega))=$ $\left.U(\omega)\right|_{O}$.

We refer the reader to [12].

4.3. Limiting Behavior of the Solution (See $[8,10])$. Take $W_{\varepsilon}=$ $\left(W(\omega) * \chi_{\varepsilon}\right)$. We have $E\left(W_{\varepsilon}\right)=0$ and $V\left(W_{\varepsilon}\right)=\sigma_{\varepsilon}^{2}=\left\|\chi_{\varepsilon}\right\|_{L^{2}(\mathbb{R})}^{2}$. Then, the variance of $W_{\varepsilon}$ tends to infinity as $\varepsilon$ tends to 0 . That implies the following.

Theorem 18. There is a subsequence $\varepsilon_{k} \rightarrow 0$ such that, $\mu$ almost surely in $\omega \in \Omega$,

$$
\begin{aligned}
\lim _{k \rightarrow 0}\left|R_{V}\left(\left(\varepsilon_{k}, \eta\right),(x, y), \omega\right)\right|=\lim _{k \rightarrow 0}\left|V(\omega)_{\varepsilon_{k}, \eta}(x, y)\right| \\
\quad=\infty
\end{aligned}
$$

for almost all $(x, y) \in \mathbb{R}^{2}$.
Proof. See [8] Corollary 1 and [10].

Assume that $\lim _{|z| \rightarrow \infty} F(z)=L$. Define the function $M$ : $\mathbb{R}^{2} \rightarrow \mathbb{R}$ by $M(x, y)=x y L$.

Theorem 19. Under the assumptions above, every subsequence of $\varepsilon \rightarrow 0$ has a subsequence $\varepsilon_{k} \rightarrow 0$ such that for all compact set $K \Subset \mathbb{R}^{2}$

$$
\begin{aligned}
\lim _{k \rightarrow 0} & \| R_{U}\left(\left(\varepsilon_{k}, \eta\right),(\cdot, \cdot), \omega\right)-R_{V}\left(\left(\varepsilon_{k}, \eta\right),(\cdot, \cdot), \omega\right) \\
& -M \|_{L^{1}(K)}=0
\end{aligned}
$$

$\mu$-almost surely.

That is,

$$
\lim _{k \rightarrow 0}\left\|U(\omega)_{\varepsilon_{k}, \eta}-V(\omega)_{\varepsilon_{k}, \eta}-M\right\|_{L^{1}(K)}=0
$$

$\mu$-almost surely.

Proof. Take $\lambda=(\varepsilon, \eta)$. We have

$$
\begin{aligned}
& U(\omega)_{\lambda}-V(\omega)_{\lambda}-M=\frac{\partial^{2} U(\omega)_{\lambda}}{\partial x \partial y} \\
& \quad-\frac{\partial^{2}\left(V(\omega)_{\lambda}+M\right)}{\partial x \partial y}, \\
& \frac{\partial^{2} U(\omega)_{\lambda}}{\partial x \partial y}-\frac{\partial^{2}\left(V(\omega)_{\lambda}+M\right)}{\partial x \partial y}=F\left(U(\omega)_{\lambda}\right) \\
& \quad-F\left(V(\omega)_{\lambda}+M\right)+\left(F\left(V(\omega)_{\lambda}+M\right)-L\right) \\
& \quad=\left(U(\omega)_{\lambda}-V(\omega)_{\lambda}-M\right) \\
& \quad \cdot \int_{0}^{1} \frac{\partial F}{\partial z}\left(U(\omega)_{\lambda}+\sigma\left(U(\omega)_{\lambda}-V(\omega)_{\lambda}-M\right)\right) d \sigma \\
& \quad+\left(F\left(V(\omega)_{\lambda}+M\right)-L\right) .
\end{aligned}
$$

So

$$
\begin{aligned}
& \left\|U(\omega)_{\lambda}-V(\omega)_{\lambda}-M\right\|_{L^{1}(K)} \\
& \leq\left\|U(\omega)_{\lambda}-V(\omega)_{\lambda}-M\right\|_{L^{1}(K)}\left\|\frac{\partial F}{\partial z}\right\|_{L^{\infty}(\mathbb{R})} \\
& \quad+\left\|F\left(V(\omega)_{\lambda}+M\right)-L\right\|_{L^{1}(K)} .
\end{aligned}
$$

By Theorem 18, there is a subsequence $\varepsilon_{k} \rightarrow 0$ such that $\mu$ almost surely in $\omega \in \Omega$ almost everywhere $\left((x, y) \in \mathbb{R}^{2}\right)$, $\lim _{k \rightarrow 0}\left|V(\omega)_{\mathcal{\varepsilon}_{k}, \eta}(x, y)\right|=\infty$, as $\lim _{|z| \rightarrow \infty} F(z)=L$ we deduce that

$$
\lim _{k \rightarrow 0}\left\|F\left(V(\omega)_{\varepsilon_{k}, \eta}\right)_{\lambda}+M-L\right\|_{L^{1}(K)}=0
$$

almost everywhere. Hence, by Lebesgue's theorem and Gronwall's lemma, the assertion follows. 
Theorem 20. Let $V \in \mathscr{D}_{\Omega}^{\prime}\left(\mathbb{R}^{2}\right)$ be the distributional solution to the free equation $\left(P_{2}\right)$. Then, the representative $U(\omega)_{\varepsilon, \eta}$ of the generalized solution to the nonlinear problem $\left(P_{1}\right)$ converge to $V+M$ with respect to the strong topology of $\mathscr{D}^{\prime}\left(\mathbb{R}^{2}\right)$, in probability as $\varepsilon \rightarrow 0$.

Proof. Let $q$ be one of the defining seminorms of the strong topology of $\mathscr{D}^{\prime}\left(\mathbb{R}^{2}\right)$. According to Theorem 19 , every subsequence of $\varepsilon \rightarrow 0$ has a subsequence $\varepsilon_{k} \rightarrow 0$ such that for all compact set $K \Subset \mathbb{R}^{2}$

$$
q\left(R_{U}\left(\left(\varepsilon_{k}, \eta\right),(\cdot, \cdot), \omega\right)-R_{V}\left(\left(\varepsilon_{k}, \eta\right),(\cdot, \cdot), \omega\right)-M\right) \longrightarrow 0
$$

almost surely. This is equivalent to convergence in probability.

\section{Conclusion}

We can use efficiently the $(\mathscr{C}, \mathscr{E}, \mathscr{P})$-algebras in studying stochastic differential equations and the white noise. We hope that this study will convince the reader that the $(\mathscr{C}, \mathscr{E}, \mathscr{P})$ algebras are a very good tool to study stochastic generalized processes.

\section{Competing Interests}

The author declares that there is no conflict of interests regarding the publication of this paper.

\section{References}

[1] J.-A. Marti, "Fundamental structures and asymptotic microlocalization in sheaves of generalized functions," Integral Transforms and Special Functions, vol. 6, no. 1-4, pp. 223-228, 1998.

[2] J.-A. Marti, "Multiparametric algebras and characteristic cauchy problem," in Non-Linear Algebraic Analysis and Applications: Proceeding of the International Conference on Generalized functions (ICGF 2000), pp. 181-192, CSP, 2004.

[3] A. Delcroix, V. Dévoué, and J.-A. Marti, "Well-posed problems in algebras of generalized functions," Applicable Analysis, vol. 90, no. 11, pp. 1747-1761, 2011.

[4] J.-F. Colombeau, New Generalized Functions and Multiplication of Distributions, vol. 84 of North-Holland Mathematics Studies, North-Holland, Oxford, UK, 1984.

[5] J.-F. Colombeau, Elementary Introduction to New Generalized Functions, vol. 113 of North-Holland Mathematics Studies, North-Holland Publishing, Amsterdam, The Netherlands, 1984.

[6] V. Dévoué, "On generalized solutions to the wave equation in canonical form," Dissertationes Mathematicae, vol. 443, pp. 169, 2007.

[7] V. Dévoué, "Generalized solutions to a non Lipschitz Goursat problem," Differential Equations \& Applications, vol. 1, no. 2, pp. 153-178, 2009.

[8] M. Oberguggenberger and F. Russo, "Nonlinear stochastic wave equations," Integral Transforms and Special Functions, vol. 6, no. 1-4, pp. 71-83, 1998.

[9] M. Nedeljkov and D. Rajter, "A note on a one-dimensional nonlinear stochastic wave equation," Novi Sad Journal of Mathematics, vol. 32, no. 1, pp. 73-83, 2002.

[10] M. Oberguggenberger and F. Russo, "Singular limiting behavior in nonlinear stochastic wave equations," in Stochastic Analysis and Mathematical Physics, vol. 50 of Progress in Probability, pp. 87-89, Springer, 2001.

[11] M. Nedeljkov and D. Rajter, "Nonlinear stochastic wave equation with Colombeau generalized stochastic processes," Mathematical Models \& Methods in Applied Sciences, vol. 12, no. 5, pp. 665-688, 2002.

[12] V. Dévoué, "Generalized solutions to a non Lipschitz-Cauchy problem," Journal of Applied Analysis, vol. 15, no. 1, pp. 1-32, 2009.

[13] J.-A. Marti, “( $\mathscr{C}, \mathscr{E}, \mathscr{P})$-sheaf structure and applications," in Nonlinear Theory of Generalized Functions, M. Grosser and Alii, Eds., Research Notes in Mathematics, pp. 175-186, Chapman \& Hall/CRC, New York, NY, USA, 1999. 


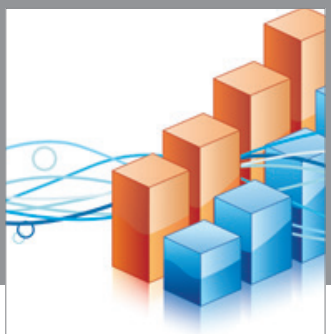

Advances in

Operations Research

vatem alat4

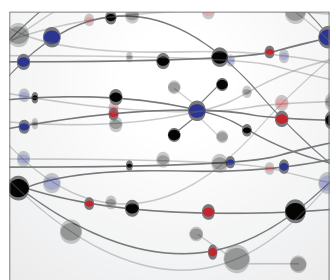

\section{The Scientific} World Journal
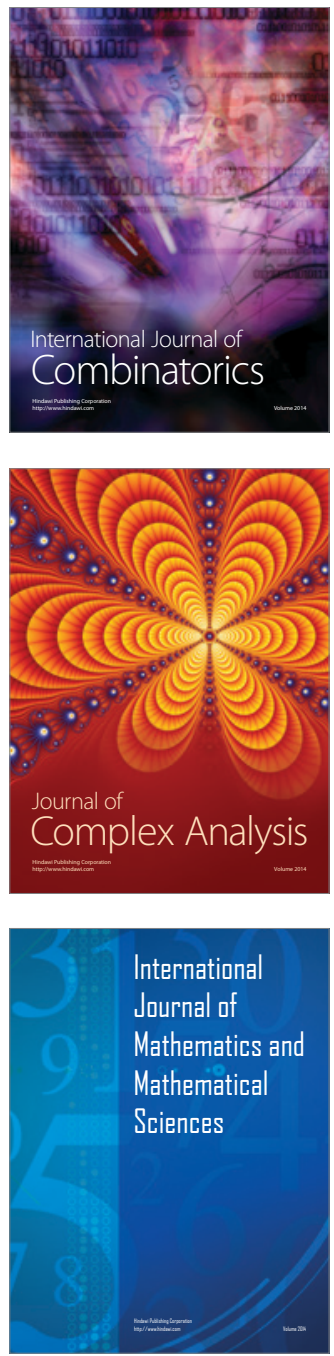
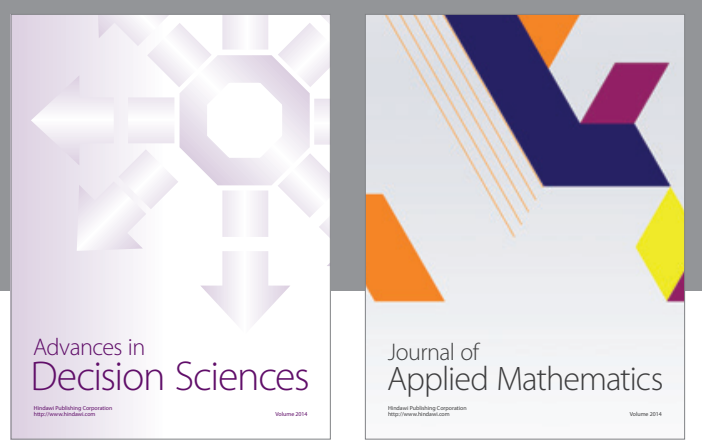

Algebra

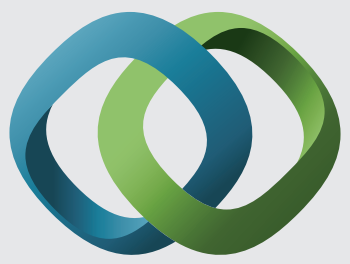

\section{Hindawi}

Submit your manuscripts at

http://www.hindawi.com
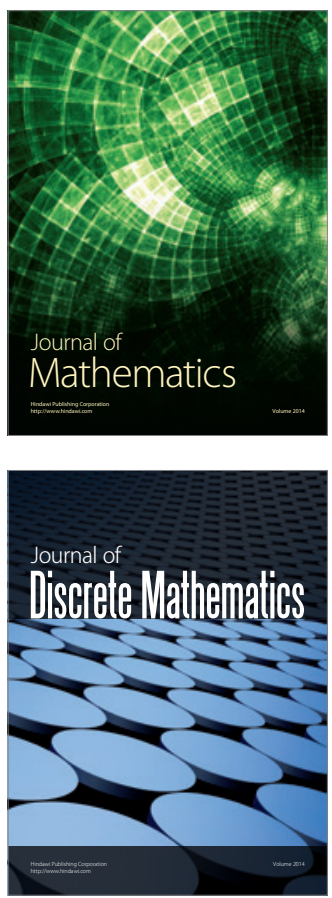

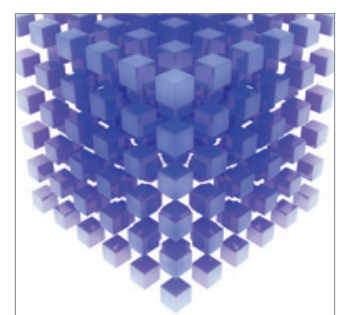

Mathematical Problems in Engineering
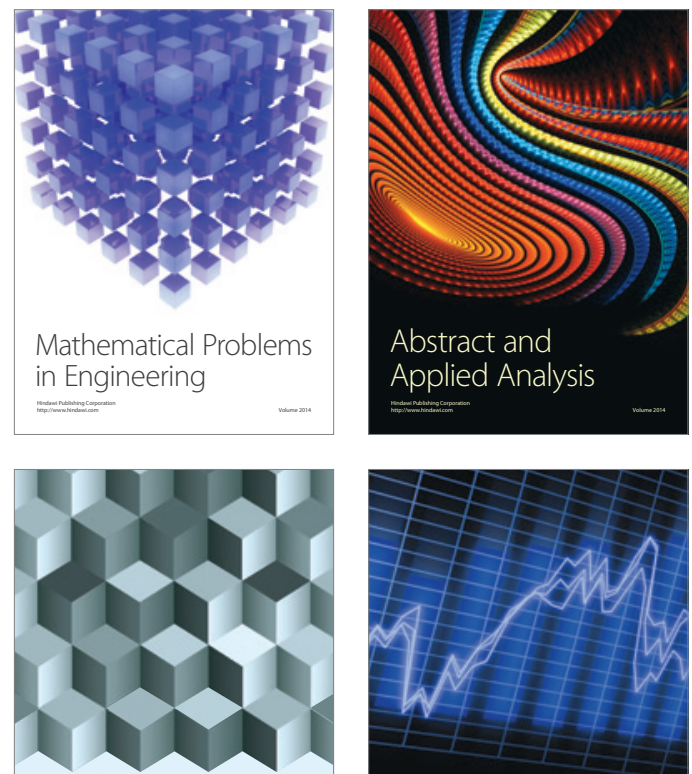

Journal of

Function Spaces

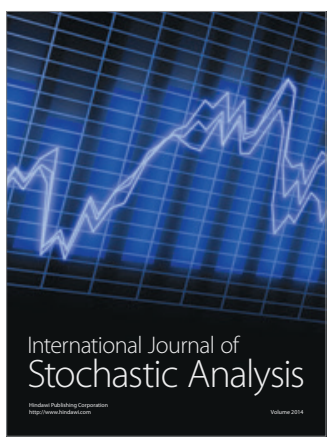

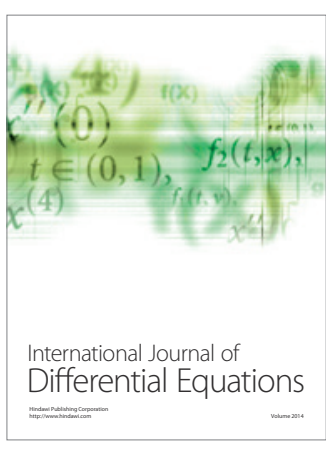
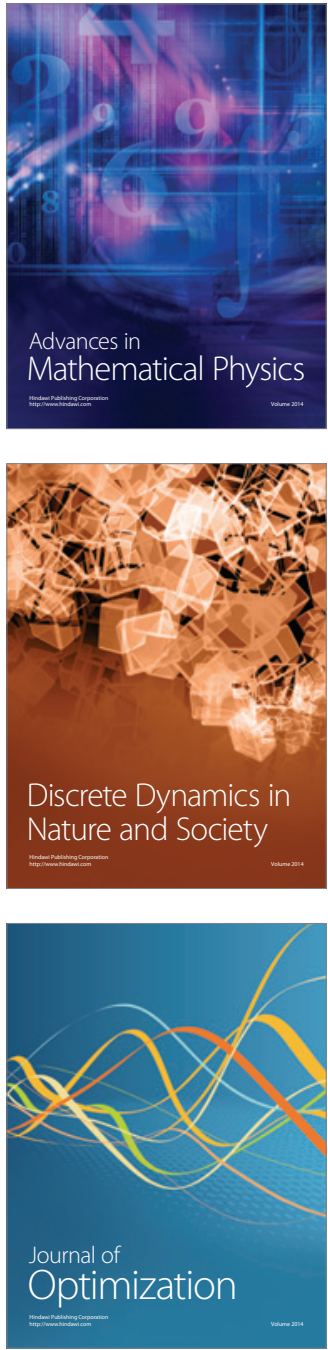